\title{
Uncertainties in (E)UV model atmosphere fluxes (Research Note)
}

\author{
T. Rauch \\ Institute for Astronomy and Astrophysics, Kepler Center for Astro and Particle Physics, Eberhard Karls University, Sand 1, \\ 72076 Tübingen, Germany \\ e-mail: rauch@astro.uni-tuebingen.de
}

Received 21 January 2008 / Accepted 4 February 2008

\section{ABSTRACT}

\begin{abstract}
Context. During the comparison of synthetic spectra calculated with two NLTE model atmosphere codes, namely TMAP and TLUSTY, we encounter systematic differences in the EUV fluxes due to the treatment of level dissolution by pressure ionization.

Aims. In the case of Sirius B, we demonstrate an uncertainty in modeling the EUV flux reliably in order to challenge theoreticians to improve the theory of level dissolution.

Methods. We calculated synthetic spectra for hot, compact stars using state-of-the-art NLTE model-atmosphere techniques.

Results. Systematic differences may occur due to a code-specific cutoff frequency of the H I Lyman bound-free opacity. This is the case for TMAP and TLUSTY. Both codes predict the same flux level at wavelengths lower than about $1500 \AA$ for stars with effective temperatures $\left(T_{\text {eff }}\right)$ below about $30000 \mathrm{~K}$ only, if the same cutoff frequency is chosen.

Conclusions. The theory of level dissolution in high-density plasmas, which is available for hydrogen only should be generalized to all species. Especially, the cutoff frequencies for the bound-free opacities should be defined in order to make predictions of UV fluxes more reliable.
\end{abstract}

Key words. atomic data - stars: atmospheres - stars: individual: HZ 43 - stars: individual: Sirius B - stars: white dwarfs ultraviolet: stars - X-rays: stars

\section{Introduction}

NLTE model atmosphere codes for hot, compact stars have arrived at a high level of sophistication and are successfully employed for spectral analyses, e.g. the Tübingen NLTE ModelAtmosphere Package TMAP ${ }^{1}$ (Werner et al. 2003; Rauch \& Deetjen 2003) in the case of $\operatorname{LS~V}+46^{\circ} 21$, the central star of Sh 2-216 (Rauch et al. 2007).

In the case of high-gravity stars like $\mathrm{LS} \mathrm{V}+46^{\circ} 21$ $\left(\log g=6.9\left[\mathrm{~cm} / \mathrm{s}^{2}\right], T_{\text {eff }}=95000 \mathrm{~K}\right)$ or neutron stars (e.g. Suleimanov \& Werner 2007), the consideration of the dissolution of atomic levels by plasma perturbation (for details see, e.g., Hummer \& Mihalas 1988) is crucial for a reliable model atmosphere calculation (Hubeny et al. 1994).

Beuermann et al. $(2006,2008)$ established the DA-type white dwarfs HZ43 $\left(\log g=7.9, T_{\text {eff }}=51111 \mathrm{~K}\right)$ and Sirius B $\left(\log g=8.6, T_{\text {eff }}=24897 \mathrm{~K}\right)$ as soft X-ray standards. For a crosscalibration between the Chandra LETG + HRC-S, the EUVE spectrometer and the ROSAT PSPC, pure hydrogen TMAP model atmospheres and synthetic spectra were used. In the case of Sirius B with a higher surface gravity $g(\log g=8.6)$, the level dissolution due to pressure ionization is even more efficient (Fig. 1) and thus, it is more important to consider it properly.

\section{Level dissolution}

Recently, Jelle Kaastra (priv. comm.) has drawn our attention to a deviation between TMAP and TLUSTY2 fluxes for Sirius B

\footnotetext{
${ }^{1}$ http://astro. uni-tuebingen. de/ rauch/TMAP/TMAP.html

${ }^{2}$ http://nova.astro.umd.edu
}

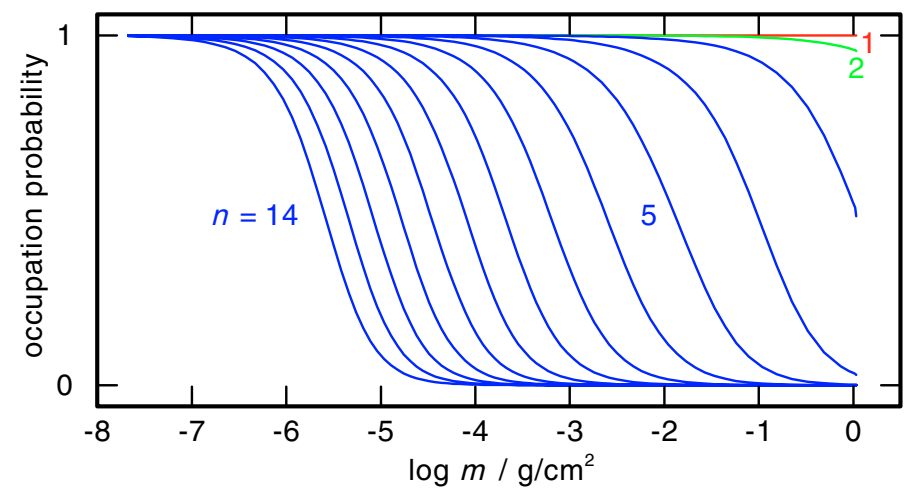

Fig. 1. Occupation probabilities of the lowest $14 \mathrm{H} \mathrm{I}$ levels considered in our TMAP model atmosphere calculation for Sirius B. Note that the line formation takes place at $\log m \approx-5.5--3.0$.

(Fig. 2) at wavelengths lower than about $1500 \AA$, while in the case of HZ 43, the model fluxes are in agreement.

In the last two decades, we have thoroughly compared TMAP and TLUSTY from time to time and found only negligible differences due to different numerical approaches and slightly different constants. In an investigation of the flux discrepancy, we are now able to identify its reason. Both codes, TMAP as well as TLUSTY, follow a generalized form to consider the level dissolution by Hubeny et al. (1994). A hitherto unsolved problem, however, is a precise, generalized formulation of the extrapolation of the hydrogen Lyman bound-free opacity into a pseudo-continuum below the unperturbed position of the 


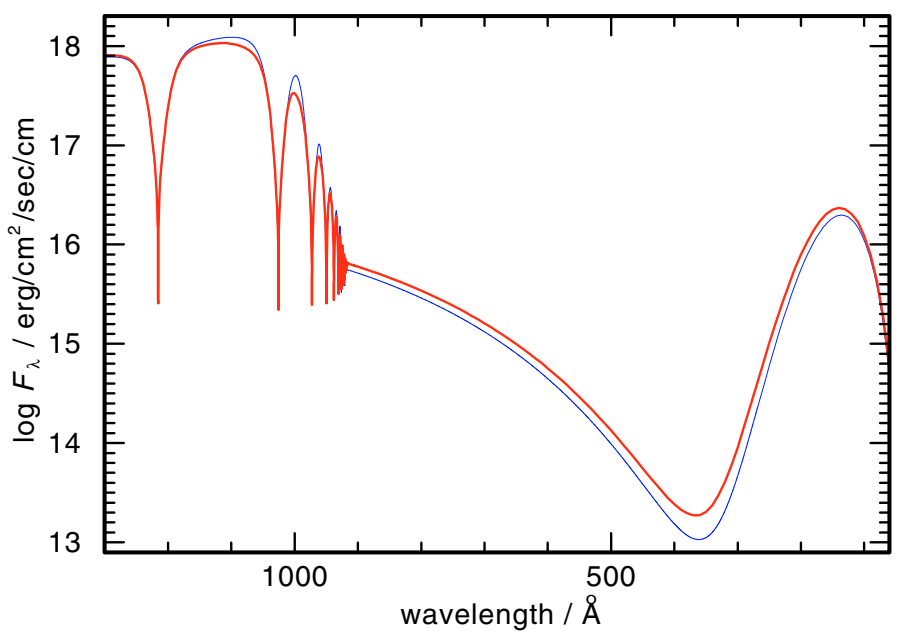

Fig. 2. Discrepancy between synthetic spectra for Sirius B calculated by TMAP (thick, long cutoff, see text) and TLUSTY (thin, short cutoff, Lanz priv. comm.) with the same parameters.

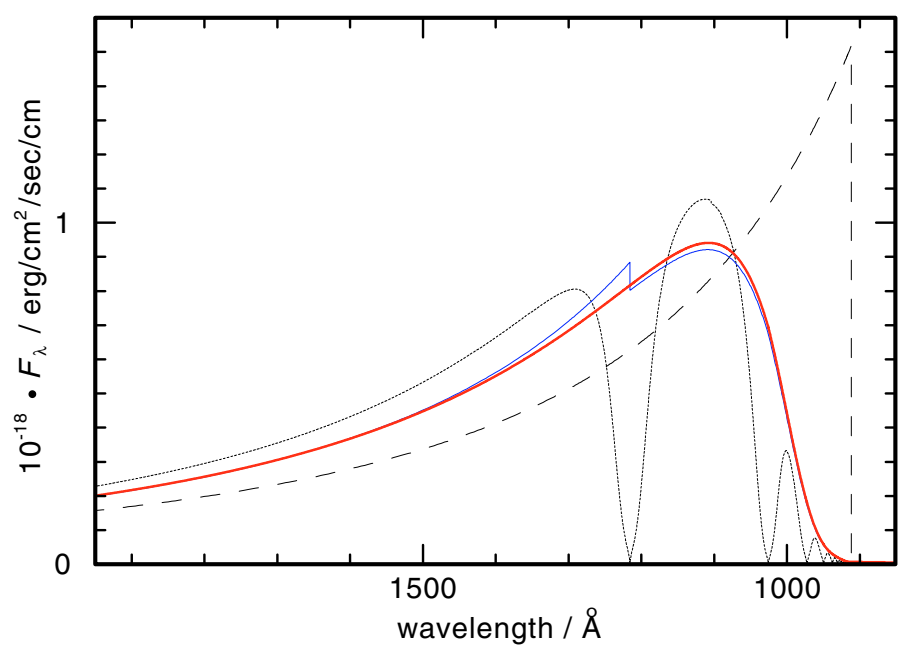

Fig. 3. Synthetic fluxes for Sirius B calculated from continuum models (no line transitions considered for test reasons) with a short (thin line) and a long cutoff (thick). The dashed line shows a synthetic spectrum with neglection of the level dissolution. Note that the artificial absorption edge at the short cutoff is hidden in the strong $\mathrm{L} \alpha$ absorption when those lines are considered (dotted).

absorption threshold (cf. Däppen et al. 1987). TMAP uses a heuristic approach with

$v_{i}^{\text {th }}=f \cdot\left[\frac{1}{n_{i}^{2}}-\frac{1}{\left(n_{i}+1\right)^{2}}\right] \cdot v_{i}^{\text {th, } 0}$

where $v_{i}^{\text {th }}$ and $v_{i}^{\text {th, }, 0}$ are the extrapolated and unperturbed threshold frequencies, respectively, of level $i$ and $n_{i}$ is its principal quantum number, i.e., $n_{i}^{2}=1$ for the Lyman continuum. Since this approach results in artificial absorption edges at $f=1$ (corresponding to $\lambda_{1}^{\text {th }}=1215.67 . \AA$, "short" cutoff), TMAP introduced $f=0.5$ (corresponding to $\lambda_{1}^{\text {th }}=2431.34 \AA$, "long" cutoff) in order to achieve a smooth transition into the continuum (Fig. 3).

TLUSTY uses a different treatment of the continuum. Based on the physical picture used to derive the pseudo-continuum formulation (cf. Däppen et al. 1987), which is valid only near the ionization limit, an artificial "short" cutoff of the boundfree opacity of the hydrogen Lyman-continuum at $\lambda_{1}^{\text {th }}=925 \AA$

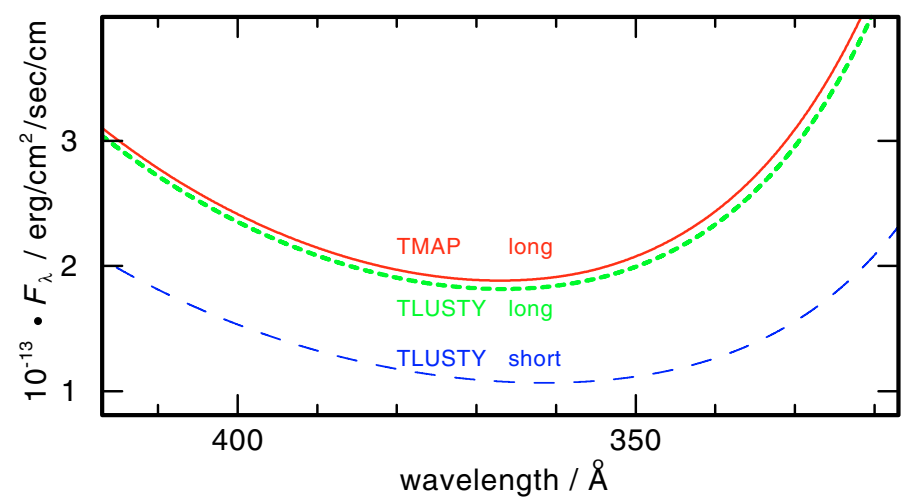

Fig. 4. Comparison of TMAP and TLUSTY (dotted: long cutoff, dashed: short cutoff) fluxes in the vicinity of the largest deviation (Fig. 2).

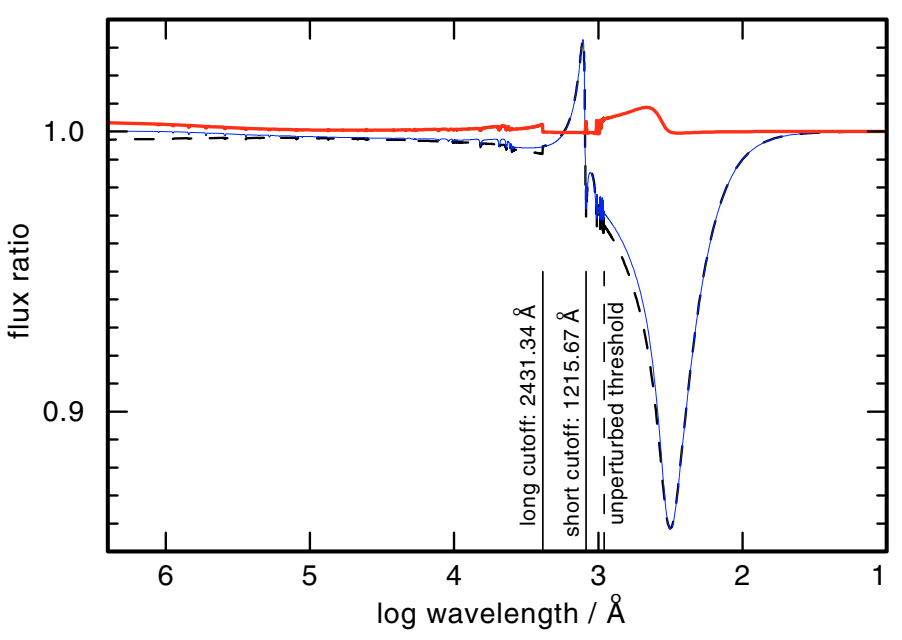

Fig. 5. Ratios of synthetic fluxes calculated by TMAP. Shown are short/long cutoff (dashed), short/extralong cutoff (thin, the extralong cutoff frequency is $10^{12} \mathrm{~Hz}$ ), and long/extralong cutoff (thick). Note that in the case of the extralong cutoff, the flux ratio slightly increases above $100000 \AA$ due to an artificial bound-free opacity in the infrared.

(Lanz priv. comm.) may be chosen (TMAP: $\lambda_{1}^{\text {th }}=2431.34 \AA$ ). A test calculation has shown that the model atmosphere fluxes of TMAP and TLUSTY agree within 5\% if a long cutoff is used by TLUSTY, too (Lanz priv. comm., Fig. 4).

Test calculations of pure hydrogen models with TMAP via the WWW interface $T M A W^{3}$ at $\log g=8.6$ and $T_{\text {eff }}$ between 10000 and $50000 \mathrm{~K}$ have shown that deviations between short-cutoff and long-cutoff model fluxes are negligible at $T_{\text {eff }} \gtrsim 30000 \mathrm{~K}$ due to the increasing degree of ionization. This explains the good agreement of TMAP and TLUSTY fluxes in case of HZ 43.

\section{Conclusions}

Since no reliable theory is available, the choice of different cutoff frequencies of the $\mathrm{H}$ I Lyman bound-free opacity in the NLTE model-atmosphere codes TMAP and TLUSTY demonstrates that the estimate of the pseudo-continuum at longer wavelengths is presently an uncertainty and definitely deserves further investigation. However, the necessity of a cutoff in order to avoid an unrealistic opacity in the infrared is shown in Fig. 5.

Moreover, a reliable theory for level dissolution by pressure ionization is presently available only for H I. Since this is

3 http://astro.uni-tuebingen.de/ rauch/TMAW/TMAW.html 
important for all other species as well, a generalized theory is highly desirable. However, this is out of the scope of this work.

Acknowledgements. We are indebted to Jelle Kaastra, Thierry Lanz and Ivan Hubeny who originally found discrepancies in the X-ray/EUV calibrations of Beuermann et al. (2006) between TLUSTY and TMAP model-atmosphere fluxes. They informed Klaus Beuermann about this issue ahead of their publication. This initiated a re-investigation of our flux calibration (Beuermann et al. 2006). We found two trivial errors (Beuermann et al. 2008) and a strong systematic difference in the case of Sirius B. Special thanks go to Thierry Lanz who then calculated two TLUSTY models with different cutoff frequencies of the H I Lyman bound-free opacity and thus worked out the basic reason for the differences between TLUSTY and TMAP.

We thank Klaus Beuermann, Klaus Werner, and Valery Suleimanov for comments and discussions.

T.R. is supported by the German Astrophysical Virtual Observatory project of the German Federal Ministry of Education and Research (BMBF) under grant 05 AC6VTB.

\section{References}

Beuermann, K., Burwitz, V., \& Rauch, T. 2006, A\&A, 458, 541 Beuermann, K., Burwitz, V., \& Rauch, T. 2008, A\&A, 481, 769

Däppen, W., Anderson, L., \& Mihalas, D. 1987, ApJ, 319, 195

Hubeny, I., \& Lanz, T. 2003, in Stellar Atmosphere Modeling, ed. I. Hubeny, D. Mihalas, \& K. Werner, ASP Conf. Ser., 288, 51

Hubeny, I., Hummer, D. G., \& Lanz, T. 1994, A\&A, 282, 151

Hummer, D. G., \& Mihalas, D. 1988, ApJ, 331, 794

Rauch, T., \& Deetjen, J. L. 2003, in Stellar Atmosphere Modeling, ed. I. Hubeny, D. Mihalas, \& K. Werner, ASP Conf. Ser., 288, 103

Rauch, T., Ziegler, M., Werner, K., et al. 2007, A\&A, 470, 317

Suleimanov, V., \& Werner, K. 2007, A\&A, 466, 661

Werner, K., Dreizler, S., Deetjen, J. L., et al. 2003, in Stellar Atmosphere Modeling, ed. I. Hubeny, D. Mihalas, \& K. Werner, ASP Conf. Ser., 288, 31 